\title{
Relationship between Petri Nets and Cellular Automata for the Analysis of Flexible Manufacturing Systems
}

\author{
Irving Barragán, Juan Carlos Seck-Tuoh, and Joselito Medina \\ Universidad Autónoma del Estado de Hidalgo, Centro de Investigación Avanzada en \\ Ingeniería Industrial, Carr. Pachuca Tulancingo Km 4.5, Mineral de la Reforma \\ Hidalgo 42184, México \\ \{irvingb, jseck, jmedina\}@uaeh.edu.mx
}

\begin{abstract}
In this paper an association between Petri nets (PN) and cellular automata (CA) is proposed to analyze the global dynamics of flexible manufacturing systems (FMS). This relation is carried out taking into account the discreteness in the dynamics of both PN and CA. In particular, generalized PN as well as one-dimensional CA are used. The work consists in modeling with $\mathrm{PN}$ both a single process with a shared resource and two parallel processes with several shared resources. The $\mathrm{PN}$ models are simplified by reduction rules and then the corresponding one-dimensional CA is obtained. Finally, the global dynamics of the FMS modeled is described by using the analysis methods of CA.
\end{abstract}

Keywords: Petri nets, cellular automata, flexible manufacturing systems.

\section{Introduction}

CA are abstract dynamical systems whose evolution is discrete in space and time. As a modeling tool, CA have been applied in the study of ecological systems, chemical systems, among others [7]. In this regard, one-dimensional CA are the most studied kind of $\mathrm{CA}$ and there exists methods contrived to study their dynamics which are mainly based on diagrams. On the other hand, PN are bipartite and directed graphs whose dynamics is discrete and deterministic in the generalized case, [18]. Due to the graphical and mathematical features, PN are a powerful modeling and analytical tool for systems which are concurrent and asynchronous such as FMS, [2], [22], [24] where PN are useful both to describe the dynamics of such systems, [13], and to prevent undesirable situations such as deadlocks, [3]. Nevertheless, some problems arise in the analysis of FMS when using the classical PN analysis methods. On the one hand, such methods can be only applied to specific PN and, on the other hand, some of the analysis methods, like the coverability tree, are not able to manage large PN models due to the state-space explosion.

The association between PN and CA has been explored in [4], [5] for modeling an ecological system. In [11] a self-timed CA is modeled with high-level PN and 
in [14] self-similar PN and CA are used to simulate Turing machines. In addition, in [16], [17] is proposed the use of CA to model FMS whose dynamics is firstly described by a net which has a similar dynamics to PN. In all these works, PN derivations are used to model the corresponding systems and in some cases the classical dynamics of CA is modified. The relationship between CA and PN we propose in this paper is carried out by using generalized PN and one-dimensional $\mathrm{CA}$ in such a way that their usual dynamics is maintained. Then, we describe the global dynamics of FMS by means of the CA analysis methods which will allow a quick and simplified way to determine some dynamical features of the FMS such as reversibility and liveness without dealing with the state-space explosion.

Additionally, we consider two kinds of FMS models to exemplify the process by which the one-dimensional CA are obtained. These FMS models are based on one of the main characteristics of such systems: shared resources. The first example is about a single process which consists of a manufacturing cell with one shared resource. The second example deals with a pair of parallel processes with several shared resources. A PN model is firstly designed for the single process as well as for the pair of parallel processes by using the most common structures to model manufacturing systems. Then, the PN models are simplified by means of PN reduction rules in order to obtain homogeneous subnets in such a way that each subnet can have the same number of markings. Such subnets will represent the cells of the CA. Furthermore, the places of the reduced PN models are considered to have a fixed capacity in the number of tokens they can hold in order to facilitate the definition of the number of states of the CA. Finally, the $\mathrm{CA}$ evolution rule is determined according to the dynamics of the PN.

The paper is organized as follows: In Sect. 2 and Sect. 2.2 we give the basic concepts of CA and PN. In Sect. 3 it is explained the process by which the one-dimensional CA are obtained from the PN models of the FMS examples. In Sect. 4 the CA analysis methods are applied to describe the global dynamics of the FMS modeled. Finally, in Sect. 5 conclusions of the work are given.

\section{Basic Concepts of Cellular Automata and Petri Nets}

\subsection{Cellular Automata}

CA consist of a configuration of cells $C$, also known as the global state, where each cell can take a state from a finite set of states $\mathcal{S}$, such that $|\mathcal{S}|=k$. Particularly, in a one-dimensional CA any configuration is a linear array of cells, that is to say $C=c_{1} c_{2} \ldots c_{i} \ldots$, where each cell forms a neighborhood $\eta\left(c_{i}\right)$ of size $2 r+1$ such that $r$ is the neighborhood radius. Usually, a one-dimensional CA can be described by the well-known Wolfram's notation $(k, r)$, [20]. The dynamics of a one-dimensional CA is determined by the evolution rule $\varphi: \mathcal{S}^{2 r+1} \rightarrow \mathcal{S}$ which assigns a new state to each cell $c_{i}^{\mathrm{t}}$ of $C^{\mathrm{t}}$ which is the global state at time t. In this way, $c_{i}^{\mathrm{t}+1}=\varphi\left(\eta\left(c_{i}^{\mathrm{t}}\right)\right)$. In spite of $C$ can have an infinite number of cells, from a practical point of view the array is taken as finite, that is $C=c_{1} c_{2} \ldots c_{l}$ where $l$ is the length of the configuration. Consequently, periodic boundary conditions are taken for the cells located at the ends of the array such that $C$ is closed 
forming a ring in such a way that each cell form a neighborhood of the same size. Furthermore, since $\varphi$ transforms a configuration $C$ into $C^{\prime}$, then $\Phi: \mathcal{C} \rightarrow \mathcal{C}$ defines a global evolution rule induced by $\varphi$ where $\mathcal{C}$ is the set of all possible configurations. In this regard, let $\mathcal{A}=\left\{C \mid \Phi^{-1}\left(C^{\prime}\right)=C\right\}$ be the set of ancestors of $C^{\prime}$ such that $C^{\prime}, C \in \mathcal{C}$, then the CA is reversible if $|\mathcal{A}|=1,[6],[15]$. Nevertheless, if $|\mathcal{A}|>1$, then $G \subset \mathcal{C}$ is a set of configurations which have no ancestors and is called the Garden of Eden.

There exists some classical methods that can be used to analyze the dynamics of one-dimensional CA like the subset and pair diagrams which are used in this paper. The subset diagram is derived from the de Bruijn diagram which can be defined by $\mathrm{B}(\mathcal{B}, \mathcal{E})$ where $\mathcal{B}=\left\{\mathrm{u} \mid \mathrm{u} \in \mathcal{S}^{2 r}\right\}$ is a set of nodes and $\mathcal{E}=\{(\mathrm{u}, \mathrm{v}) \mid \mathrm{u}, \mathrm{v} \in \mathcal{B}\}$ is a set of links, [19]. In this way, let $\mathrm{u}=a x$ and $\mathrm{v}=x b$ be two nodes in the de Bruijn diagram such that $x \in \mathcal{S}^{2 r-1}$ and $a, b \in \mathcal{S}$, then $\mathrm{u}$ and $\mathrm{v}$ can be linked if there exists $\mathrm{u} \odot \mathrm{v}=a x b$, where $\odot$ defines an overlapping operation between two nodes and $a x b \in \mathcal{S}^{2 r+1}$. Thus, each link is labeled with $c \in \mathcal{S}$ such that $\varphi(a x b)=c$. Besides, an evolution matrix can be obtained from the de Bruijn diagram in such a way that the nodes constitutes the rows and the columns of the matrix and each entry is either determined by $\varphi(\mathrm{u} \odot \mathrm{v})$ if the link exists or by a dot if the link does not exist, [9].

Therefore, the subset diagram is composed of the subsets of $\mathcal{B}$. In this way, let $W, Z \subseteq \mathcal{B}$ be two nodes of the subset diagram, then $W$ and $Z$ are linked if for each node $\mathrm{w} \in W$ there exists another node $\mathrm{z} \in Z$ such that $\varphi(\mathrm{w} \odot \mathrm{z})=c$ and $c$ labels the link. Finally, the cycle diagram consists in graphing the evolution trajectory of all the $k^{l}$ possible configurations $C \in \mathcal{C}$. Hence, a given configuration $C$ can appear again in the trajectory of its evolution forming a cycle, [21]. Nevertheless, if there are configurations that do not appear in the cycle then they belong to a sequence of configurations that leads to the cycle. Such a sequence forms a branch of the cycle and, therefore it has an end which is a configuration which has no ancestors.

\subsection{Petri Nets}

A PN consists of the sets of nodes $\mathcal{P}=\left\{p_{1}, p_{2}, \ldots, p_{m}\right\}$ and $\mathcal{T}=\left\{t_{1}, t_{2}, \ldots, t_{n}\right\}$, called places and transitions, respectively [1], [12], such that $m, n \in \mathbb{Z}^{+}$where $\mathbb{Z}^{+}=\{1,2, \ldots\}$. Moreover, $\mathcal{P} \cup \mathcal{T} \neq \emptyset$ and $\mathcal{P} \cap \mathcal{T}=\emptyset$. The nodes are connected by a set of directed arcs defined by $\mathcal{F} \subseteq(\mathcal{P} \times \mathcal{T}) \cup(\mathcal{T} \times \mathcal{P})$. In addition to this, $W: \mathcal{F} \rightarrow \mathbb{N}$ is a weight function and $M_{0}: \mathcal{P} \rightarrow \mathbb{N}$ defines the initial marking, where $\mathbb{N}=\{0,1,2, \ldots\}$. In this regard, $\mathbf{N}=(\mathcal{P}, \mathcal{T}, \mathcal{F}, W)$ is the structure of a PN whereas $\left(\mathbf{N}, M_{0}\right)$ is the PN with a given initial marking, [10]. Furthermore, any marking $M$ is represented by an $m \times 1$ vector such that $\boldsymbol{M}\left(p_{i}\right)=s$ is the number of tokens of place $p_{i}$ where $i=1, \ldots, m$ and $s \in \mathbb{N}$. In this way, it is possible to associate a maximum number of tokens to each place of a PN such that, for each place $p \in \mathcal{P}, K: \mathcal{P} \rightarrow \mathbb{Z}^{+}$is the function that assigns the maximum number of tokens that a place can hold at any marking.

As for the PN dynamics, let $\bullet=\{p \mid W(p, t)>0\}$ and $t^{\bullet}=\{p \mid W(t, p)>0\}$ be the sets of input and output places of $t$, respectively. Then for a given net 
$\left(\mathbf{N}, M_{0}\right)$ its dynamics or marking change is carried out by applying the enabling and firing rule. A transition $t$ is enabled at the marking $M_{d}(d \in \mathbb{N})$ if $\forall p \in \bullet t$, $M_{d}(p) \geq W(p, t)$ and, in the case of PN of finite capacity, $\forall p \in t^{\bullet}, M_{d}(p) \leq$ $K(p)-W(t, p)$. As a result, if $t$ is enabled at the marking $M_{d}$ then $t$ can be fired transforming $M_{d}$ into $M_{d+1}$ such that $M_{d+1}(p)=M_{d}(p)-W(p, t)+W(t, p)$.

PN properties are classified into dynamical and structural ones. Dynamical properties are those which depends on the initial marking whereas structural properties are independent of the initial marking. In this paper we are interested in some dynamical properties which are reachability, reversibility and liveness. In this regard, let $\mathcal{M}\left(M_{0}\right)$ be the set of reachable markings of a PN from $M_{0}$ and $\mathcal{L}\left(M_{0}\right)$ be the set of firing sequences executable from $M_{0}$. Then, a marking $M_{q}$ is reachable from $M_{0}$ if there exists a firing sequence $\sigma=t_{1} t_{2} \cdots t_{q}$ that leads to $M_{q}$. This is denoted by $M_{0} \stackrel{\sigma}{\rightarrow} M_{q}$, such that $\sigma \in \mathcal{L}\left(M_{0}\right)$. In this way, a PN is reversible if for each marking $M \in \mathcal{M}\left(M_{0}\right), M_{0}$ is reachable from $M$. A PN is live if $\forall t \in \mathcal{T}$ and for any marking $M \in \mathcal{M}\left(M_{0}\right), t$ appears in some sequence $\sigma \in \mathcal{L}\left(M_{0}\right)$ such that $M_{0} \stackrel{\sigma}{\rightarrow} M,[18]$.

Among the basic methods that can be used to analyze a $\mathrm{PN}$, reduction (or augmentation) rules are procedures to simplify PN with a complex structure but keeping some dynamical properties like liveness. The following reduction/augmentation rules are the basic ones, [23]:

Rule 1. Reduction/augmentation of series places.

Rule 2. Reduction/augmentation of series transitions.

Rule 3. Reduction/augmentation of parallel places.

Rule 4. Reduction/augmentation of parallel transitions.

Rule 5. Elimination/addition of self-loop places.

Rule 6. Elimination/addition of self-loop transitions.

Dynamical properties such as reachability, liveness as well as reversibility are of special interest for modeling and analyzing the FMS performance. For instance, liveness is a sign of absence of deadlocks which arise from the competition for the access to shared resources. Reachability can be used during the design stage to identify whether the modeled system can reach a particular state and reversibility is connected with error recovery.

\section{Construction of One-Dimensional CA from PN Models}

In this section we explain how to obtain a one-dimensional CA from PN models of FMS. In order to carry out this task, two examples of FMS are considered. These examples comprise the most common structures and features of FMS. The first example is a manufacturing cell, shown in Fig. 1 which is composed of a robot which is shared by three machines named M1, M2 and M3. The second example consists of two parallel processes where two types of parts can be machined, Parts A and Parts B. In order to perform the processing of both kinds of parts, machines M1, M2, M3, M4 and M5 must be shared as is illustrated in Fig. 2. 


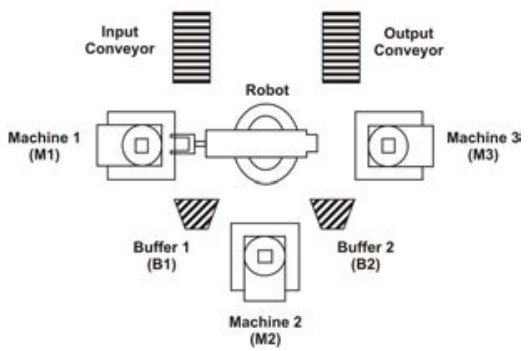

Fig. 1. A manufacturing cell with one shared resource

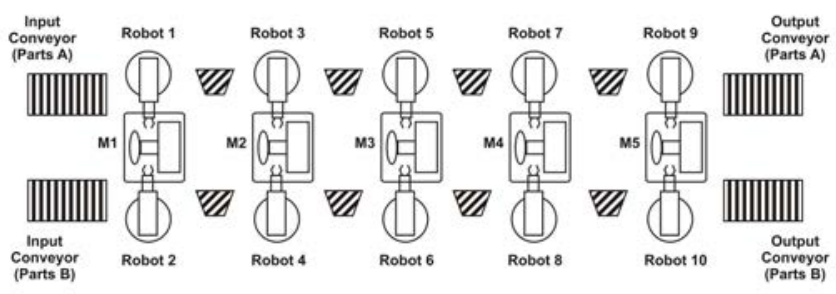

Fig. 2. Parallel processes with five shared resources

The PN models of the corresponding examples of FMS and their initial markings are depicted in Fig. 3 and Fig. 4, correspondingly. We consider that only one part can be available at the beginning of each process in such a way that any other can arrive until the first one leaves the system.

In order to obtain a one-dimensional $\mathrm{CA}$ from each $\mathrm{PN}$ model, the reduction rules 2 and 5 shown in Figs. 5a and 5b, respectively, are applied to such PN models. The reason to simplify the PN models is that it is desired to obtain homogeneous subnets to form the cells of the automaton and, according to the number of possible markings of each subnet, the states of the CA can be specified. In this regard, to facilitate the definition of the number of states of the CA, the places of the reduced PN models are limited to hold one token such that for any place $p, K(p)=1$. Furthermore, the neighborhood radius for each CA obtained from the reduced $\mathrm{PN}$ is taken as $r=1$.

\subsection{Example 1: Obtaining a One-Dimensional CA for a Single Process in Series}

A first reduction for the $\mathrm{PN}$ shown in Fig. 3 is carried out by applying the Rule 2 , and the result is shown in Fig. 6 a. Then, the place $p_{16}$ is eliminated by Rule 5 , obtaining the net shown in Fig. $6 \mathrm{~b}$. From this net it can be obtained two kinds of subnets. One subnet has one place and the other one has two places. In this way, these subnets constitutes the cells of the CA as it is shown in Fig. 7, whereas the possible markings in each subnet determine the number of states of the CA. 


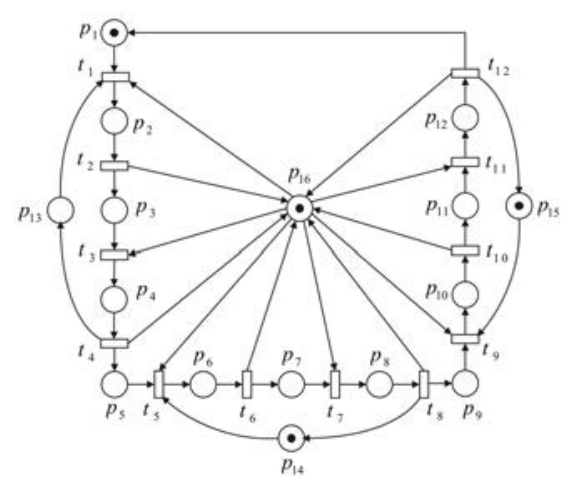

Fig. 3. PN model of the manufacturing cell with a shared resource

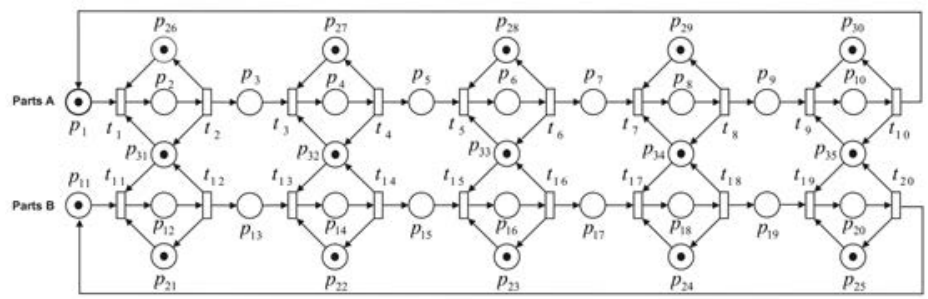

Fig. 4. PN model of the parallel processes with five shared resources

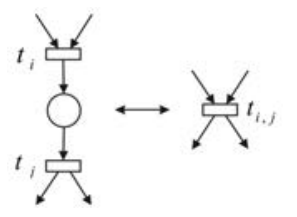

(a)

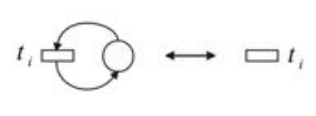

(b)

Fig. 5. (a) Rule 2: Reduction/augmentation of series transitions; (b) Rule 5: Elimination/addition of self-loop places

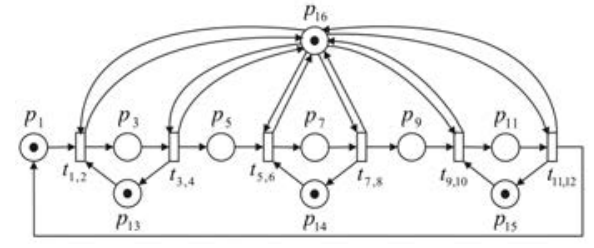

(a)

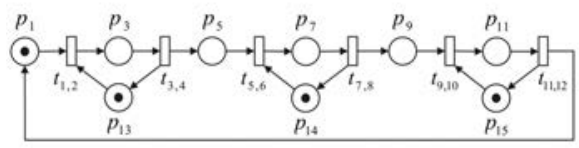

(b)

Fig. 6. Reductions of the PN of Fig. 3 by applying (a) Rule 2 and (b) Rule 5 
The CA states are taken as 0 and 1 , such that $\mathcal{S}=\{0,1\}$. Hence, for subnets with one place the state is 0 whenever the place is not marked, which means that there is no available parts, and 1 if the place is marked. For subnets with two places take as example the one which has the places $p_{13}$ and $p_{3}$. Thus, when $p_{3}$ is marked it means that the corresponding operation is being performed and thus the state is taken as 1 . In contrast to this, when place $p_{13}$ is marked the state is 0 which means that the operation is not being executed. The evolution rule $\varphi$ is determined according to the dynamics of the PN of Fig. 7. In other words, consider $\eta\left(c_{2}\right)=c_{1} c_{2} c_{3}$, since $p_{3}$ and $p_{13}$ are related to $p_{1}$ and $p_{5}$ by transitions $t_{1,2}$ and $t_{3,4}$ respectively, then if either $t_{1,2}$ or $t_{3,4}$ are fired they will cause a marking change of $p_{3}$ and $p_{13}$ and, as a result, $c_{2}$ change its state. In this way, $\varphi$ is described by the evolution matrix defined in Equation (1). Thus, the PN of Fig. 7 is simulated by a CA $(2,1)$ where the initial configuration is

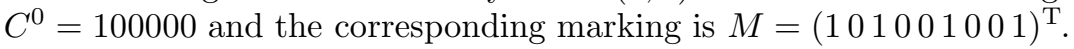

00
01
10
11 $\left[\begin{array}{cccc}0 & 0 & 10 & 11 \\ \cdot & \cdot & 1 & \cdot \\ 1 & 1 & \cdot & \cdot \\ \cdot & \cdot & 0 & 1\end{array}\right]$

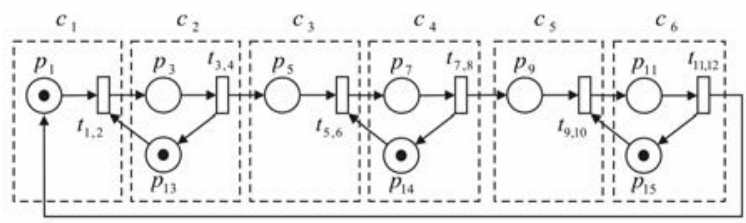

Fig. 7. Cells for the one-dimensional CA which simulates the PN of Fig. 6b

\subsection{Example 2: Obtaining a One-Dimensional CA for Processes in Parallel.}

In a similar way to Example 1, the PN model of Fig. 4 is reduced by firstly applying the Rule 2 and then the Rule 5 such that the result is shown in Fig. 8. The subnets that form the cells of the CA are depicted in Fig. 9 from which it is observed that there can be four possible markings for each subnet.

Thus, the set of states of the CA is defined by $\mathcal{S}=\{0,1,2,3\}$ and the correspondence between the markings and the states is described in Table 1, taking as example the subnet with the places $p_{1}, p_{11}, p_{31}$ which represent the cell $c_{1}$. With respect to the $\mathrm{PN}$ dynamics, it is established that whenever occurs a conflict for the access to a shared resource, Parts A must be processed first. Moreover, the enabled transitions are fired at the same time if the places do not exceed the capacity of one token and if such transitions are not in conflict. Considering this, the evolution rule is defined according to the marking change of the PN and is 


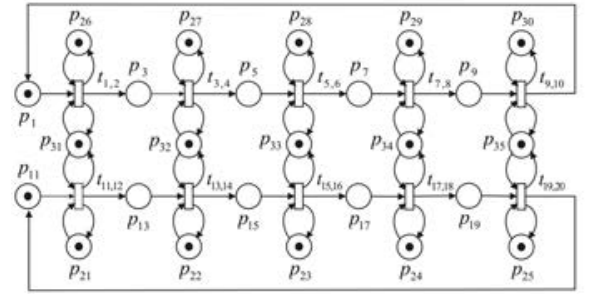

(a)

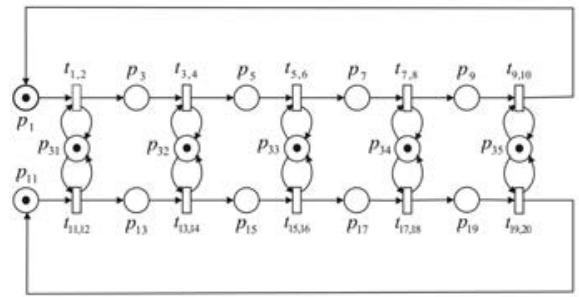

(b)

Fig. 8. Reductions for the PN model of Fig. 4 by applying (a) Rule 2 and (b) Rule 5

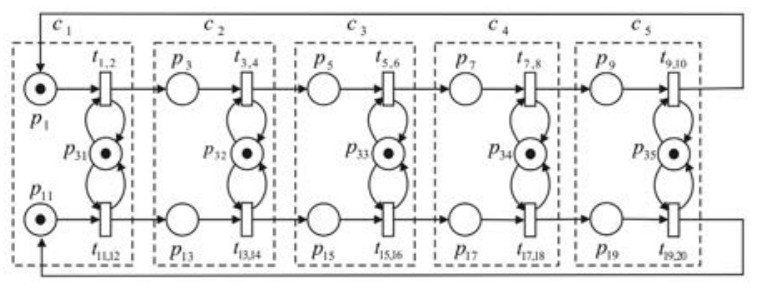

Fig. 9. Cells for the CA $(4,1)$ that represents the dynamics of the processes in parallel

defined by the evolution matrix shown in Equation (2). Hence, a CA $(4,1)$ is used to simulate the PN of Fig. 9 where the initial configuration is $C^{0}=30000$.

$$
\begin{aligned}
& \begin{array}{llllllllllllllll}
00 & 01 & 02 & 03 & 10 & 11 & 12 & 13 & 20 & 21 & 22 & 23 & 30 & 31 & 32 & 33
\end{array}
\end{aligned}
$$

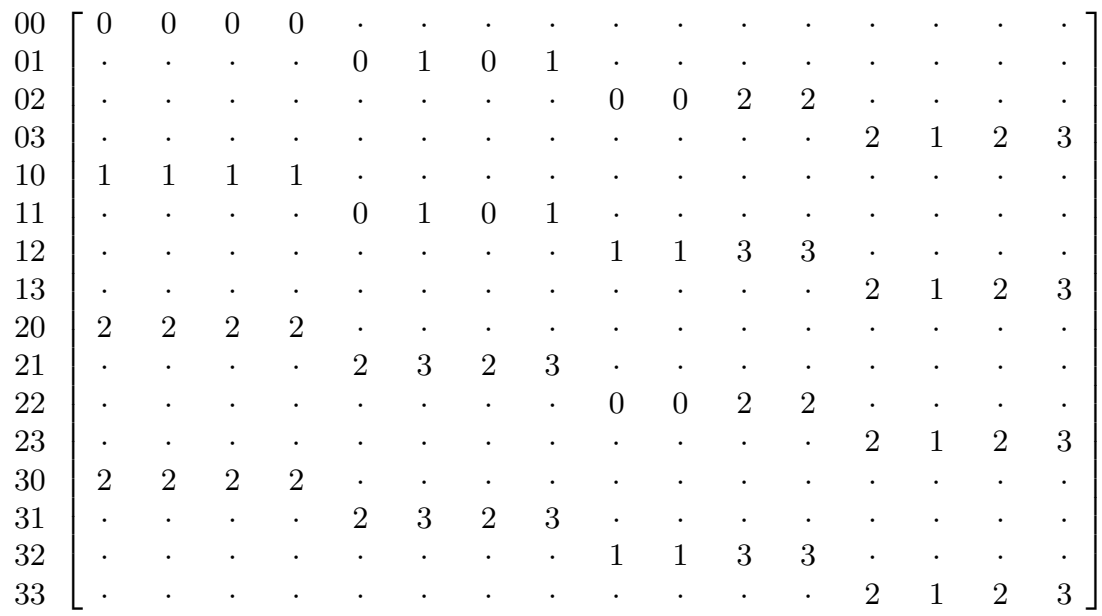


Table 1. States that can take each cell according to the markings of each subnet of Fig. 9, taking the possible markings of $p_{1}, p_{31}$ and $p_{11}$ as examples.

\begin{tabular}{cccc}
\hline$M\left(p_{1}\right)$ & $M\left(p_{31}\right)$ & $M\left(p_{11}\right)$ & State \\
\hline 0 & 1 & 0 & 0 \\
0 & 1 & 1 & 1 \\
1 & 1 & 0 & 2 \\
1 & 1 & 1 & 3 \\
\hline
\end{tabular}

\section{Applying the CA Analysis Methods to Describe the FMS Dynamics}

The analysis of the global dynamics of the PN models of Figs. 7 and 9 is carried out by means of the CA analysis methods explained in Sect. 2.2. These diagrams were obtained with the help of the NXLCAU software developed by $\mathrm{H}$. V. McIntosh, [8].

\subsection{Analysis of the FMS of Example 1}

In Fig. 10a is shown the subset diagram of the CA $(2,1)$ that simulates the PN of Fig. 7. From this diagram it is possible to identify those configurations that can be formed or not in the CA. For instance, if there is a path from node 15 to node 0 , that is to say, from the full set to the empty set, then the formed configuration does not have any ancestors and it only can appear as an initial configuration.

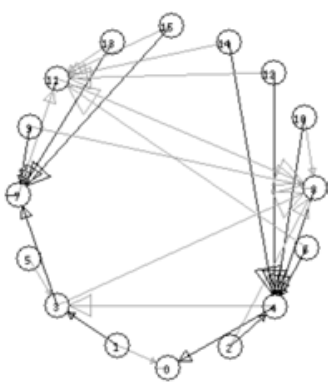

(a)

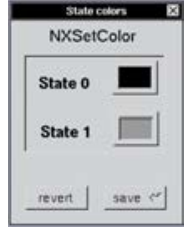

(b)

Fig. 10. (a) Subset diagram for the CA $(2,1)$ of Example 1. (b) State color settings of NXLCAU for the subset diagram.

In this sense, the path $15 \stackrel{1}{\rightarrow} 11 \stackrel{1}{\rightarrow} 11 \stackrel{0}{\rightarrow} 8 \stackrel{0}{\rightarrow} 4 \stackrel{0}{\rightarrow} 0 \stackrel{1}{\rightarrow} 0$ forms a configura-

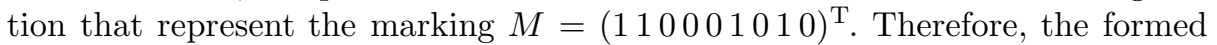
configuration belongs to the Garden of Eden and in the PN the corresponding marking cannot be formed because of the places of the PN can have at most one 
token and all the enabled transitions are fired simultaneously, if they are not in conflict. Even though there are configurations that can be formed in the CA according to the subset diagram, these configurations represent markings that are undesirable in the PN. For example, the marking $M=(111111111)^{\mathrm{T}}$ is a deadlock since any transition can be fired, considering that the $\mathrm{PN}$ is of finite capacity. In the CA evolution the corresponding configuration leads to a cycle of only one node as it is shown in Fig. 11a.

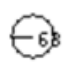

(a)

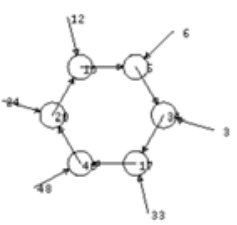

(b)

Fig. 11. Cycle diagrams of the CA $(2,1)$ with $l=6$

Cycle diagrams are useful for determining which configurations are part of a cycle or even which configurations lead to a cycle and in how many steps. For example, Fig. 11b shows a cycle diagram where nodes 10, 5, 34, 17, 40, 20, 10 form the cycle and nodes 12, 6, 3, 33, 48 and 24 lead to the cycle in one step. That is to say, the configuration $C=000110$ (node 6 ), which represents

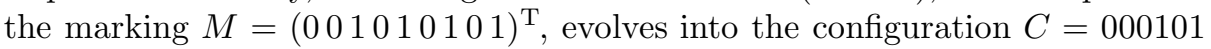

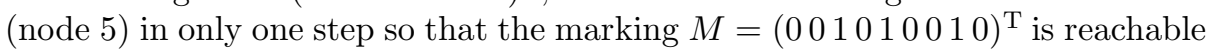

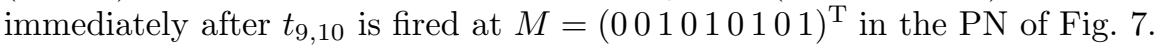

\subsection{Analysis of the FMS of Example 2}

Cycle diagrams are used here in order to perform the analysis of the PN since it is not possible to show the subset diagram because of its size. In this way, Fig. 12 shows all the cycle diagrams that are obtained for the CA $(4,1)$. It can be observed from Fig. 12 that the first four cycle diagrams (from the left upper corner of the figure to the right) are composed of only one node, which means that the corresponding markings in the PN are deadlocks since the evolution of the $\mathrm{CA}$ as well as the marking change in the PN remain unalterable. Similarly,

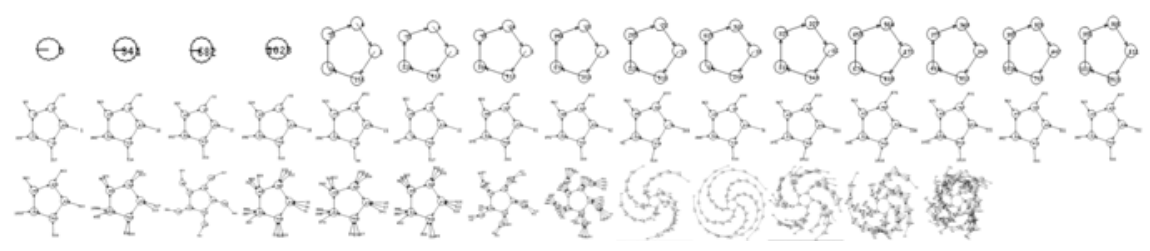

Fig. 12. Cycle diagrams for the CA $(4,1)$ of Example 2 with $l=5$ 
for the next eleven cycle diagrams, which are formed by a cycle of five nodes, each of the configurations of the cycles can be initial markings in the PN such that for these markings the PN is reversible.

\section{Conclusions}

In this paper a relationship between PN and CA was presented in order to carry out the analysis of the global dynamics of FMS. This association was carried out by using generalized PN and one-dimensional CA. Two examples of FMS were considered to illustrate the process by which CA analysis methods are applied to the study of the dynamics of such FMS. This examples consisted in a single process as well as in a pair of parallel processes both with shared resources which is a common feature of FMS. PN models were designed for such FMS examples and then such PN models were simplified by means of PN reduction rules in order to obtain the corresponding one-dimensional CA. Then, by using $\mathrm{CA}$ analysis methods, mainly subset and cycle diagrams, the PN models were analyzed to determine which configurations can be formed in the $\mathrm{CA}$ and therefore which markings can be reached in the PN under the dynamical conditions imposed to them. In this way, it could be determined, according to the characteristics of the CA dynamics, some features of the FMS like the absence of deadlocks and reversibility. On the whole, CA provided a simplified analysis of the global dynamics of FMS by obtaining one-dimensional CA from the PN models. In spite of that, not all configurations in the evolution of CA are significant markings in the PN dynamics, in the sense that they could not have a practical meaning in the system modeled. Nevertheless, an advantage of the use of CA analysis methods is that, no matter what initial configuration is taken in the PN, it can be determined which will be the dynamics in a global way. Future studies about the analysis of PN dynamics by means of the relationship between PN and CA proposed here will be carried out in depth in a next paper.

\section{References}

1. David, R., Alla, H.: Petri Nets for Modeling of Dynamic Systems-A survey. Automatica 30, 175-202 (1994)

2. D'Souza, K.A., Khator, S.: A Survey of Petri Net Applications in Modeling Controls for Automated Manufacturing Systems. Computers in Industry 24, 5-16 (1994)

3. Ezpeleta, J., Colom, J., Martínez, J.: A Petri Net Based Deadlock Prevention Policy for Flexible Manufacturing Systems. IEEE Transactions on Robotics and Automation 11, 173-184 (2002)

4. Gronewold, A., Sonnenschein, M.: Asynchronous Layered Cellular Automata for the Structured Modelling of Ecological Systems. In: Hahn, W., Lehmann, A. (eds.) 9th European Simulation Symposium (ESS 1997), pp. 286-290 (1997)

5. Gronewold, A., Sonnenschein, M.: Event-Based Modelling of Ecological Systems with Asynchronous Cellular Automata. Ecological Modelling 108, 37-52 (1998)

6. Kari, J.: Representation of Reversible Cellular Automata with Block Permutations. Mathematical Systems Theory 29, 47-61 (1996) 
7. Kari, J.: Theory of Cellular Automata: A Survey. Theoretical Computer Science 334, 3-33 (2005)

8. McIntosh, H.V.: NXLCAU software, http://delta.cs.cinvestav.mx/ mcintosh/ cellularautomata/Welcome.html

9. McIntosh, H.V.: Linear Cellular Automata Via de Bruijn Diagrams, http://delta.cs.cinvestav.mx/ mcintosh/comun/cf/debruijn.pdf

10. Murata, T.: Petri Nets: Properties, Analysis and Applications. Proceedings of IEEE 77, 541-580 (1989)

11. Penttinen, O.: Modelling and Analysis of Self-timed Cellular Automata Using HighLevel Petri Nets. In: Proc. of Workshop on Token based computing (ToBaCo), Satellite Event of the 25-th International Conference on Application and Theory of Petri Nets (2004)

12. Peterson, J.L.: Petri Nets. Computing Surveys 9, 223-252 (1977)

13. Recalde, L., Silva, M., Ezpeleta, J., Teruel, E.: Petri Nets and Manufacturing Systems: An Examples-Driven Tour. In: Desel, J., Reisig, W., Rozenberg, G. (eds.) Lectures on Concurrency and Petri Nets. LNCS, vol. 3098, pp. 742-788. Springer, Heidelberg (2004)

14. Schaller, M., Svozil, K.: Scale-Invariant Cellular Automata and Self-similar Petri Nets. The European Physical Journal B 69, 297-311 (2009)

15. Seck-Tuoh, J.C.: Matrix Methods and Local Properties of Reversible OneDimensional Cellular Automata. J. Phys. A: Math. Gen. 35, 5563-5573 (2002)

16. Shen, H., Chau, H., Wong, K.: An Extended Cellular Automaton Model for Flexible Manufacturing Systems. The International Journal of Advanced Manufacturing Technology 11, 258-266 (1996)

17. Shen, H., Yan, W.: Modeling Autonomous Assembly Systems and FMS Using Cellular Automata. The International Journal of Advanced Manufacturing Technology 7, 333-338 (1992)

18. Silva, M.: Las Redes de Petri: En la Automática y la Informática. AC, Madrid (2002)

19. Sutner, K.: De Bruijn Graphs and Cellular Automata. Complex Systems 5, 19-30 (1991)

20. Wolfram, S.: Statistical Mechanics of Cellular Automata. Reviews of Modern Physics 55, 601-644 (1983)

21. Wuensche, A., Lesser, M.: The Global Dynamics of Cellular Automata. Santa Fe Institute Studies in the Sciences of Complexity. Addison-Wesley Publishing Company, USA (1992)

22. Zhang, W., Li, Q., Zha, X.F.: A Generic Petri Net Model for Flexible Manufacturing Systems and Its Use for FMS Control Software Testing. Int. J. Prod. Res. 38, 1109-1131 (2000)

23. Zhou, M.-C., Venkatesh, K.: Modeling, Simulation and Control of Flexible Manufacturing Systems: A Petri Net Approach. World Scientific, Singapur (1999)

24. Zurawski, R., Zhou, M.C.: Petri Nets and Industrial Applications: A Tutorial. IEEE Transactions on Industrial Electronics 41, 567-583 (1994) 